\title{
Prognostic Impact of Left Atrial Minimal Volume on Clinical Outcome in Patients with Non-Obstructive Hypertrophic Cardiomyopathy
}

\author{
Sung-Hee Shin, ${ }^{1}$ MD, Ji-Hoon Jang, ${ }^{1}$ MD, Yong-Soo Baek, ${ }^{1}$ MD, Sung-Woo Kwon, ${ }^{1}$ MD, \\ Sang-don Park, ${ }^{1}$ MD, Seong-Ill Woo, ${ }^{1}$ MD, Dae-Hyeok Kim, ${ }^{1}$ MD and Jun Kwan, ${ }^{1}$ MD
}

\begin{abstract}
Summary
Maximal left atrial volume (LAVmax) has been suggested to be an important indicator of left ventricular (LV) diastolic function and a prognosticator in patients with hypertrophic cardiomyopathy (HCM). However, LAVmax can be influenced by LV longitudinal systolic function, which causes systolic descent of the mitral plane. We investigated the prognostic role of LAVmin in patients with HCM and tested if LAVmin is better than LAVmax in predicting clinical outcome in these patients. A total of 167 consecutive patients with HCM were enrolled (age $=64.7 \pm 13.5$ years, male: female $=120: 47$ ). Clinical parameters and conventional echocardiographic measurement including tissue Doppler measurement were evaluated. Left atrial maximal and minimal volumes were measured just before mitral valve opening and at mitral valve closure respectively using the biplane disk method. The relationship between LAVmin and the clinical outcome of hospitalization for heart failure (HF), stroke or all-cause mortality was evaluated. During a median follow-up of $25.0 \pm 17.8$ months, the primary end point of HF hospitalization, stroke or death occurred in 35 patients (21\%). Indexed LAVmin was predictive of HF, stroke or death after adjustment for age, diabetes, hypertension, atrial fibrillation, LV ejection fraction, and E/e'in a multivariate analysis $(P=0.001)$. The model including indexed LAVmin was superior to the model including indexed LAVmax in predicting a worse outcome in patients with $\mathrm{HCM}(P=0.02)$. In conclusion, LAVmin was independently associated with increased risk of HF, stroke, or mortality in patients with HCM and was superior to LAVmax in predicting clinical outcome in this population.
\end{abstract}

(Int Heart J 2018; 59: 991-995)

Key words: Minimal LA volume, Prognosis

$\mathrm{H}$ ypertrophic cardiomyopathy (HCM) is a common genetic heart disease and can show a diverse clinical presentation and course. ${ }^{1-3)}$ While most individuals with HCM are asymptomatic and have a normal life expectancy without significant disability, some can develop symptoms. ${ }^{4.5)}$ Sudden cardiac death and heart failure (HF) are possible serious complications that may arise in these patients. The presence of dynamic left ventricular outflow tract (LVOT) obstruction is known to be related to symptomatic conditions, development of atrial fibrillation (AF), and death. ${ }^{6.7)}$ On the other hand, patients with non-obstructive HCM appear to experience a largely stable clinical course, associated with relatively low risk of advanced HF symptoms, other disease complications, and mortality than patients with obstructive disease. ${ }^{8.9)}$ However, there is a significant subgroup of patients with non-obstructive $\mathrm{HCM}$ at risk for HF because of systolic or diastolic dysfunction and death. In addition, AF and subsequent thromboembolic events are not uncommon in this population. ${ }^{10)}$

\section{Editorial p.906}

Numerous studies have shown that maximal left atrial volume (LAV) is a strong predictor of clinical outcome in various cardiovascular diseases. ${ }^{11-13)}$ In HCM, LAV is also known to be related to exercise capacity, development of $\mathrm{AF}$, and poor prognosis. ${ }^{14-18)} \mathrm{LAV}$ can reflect the burden and chronicity of elevated left ventricular (LV) filling pressure and function as a prognosticator. However, maximal LAV (LAVmax) can be influenced by LV longitudinal systolic function, which causes systolic descent of the mitral plane. ${ }^{19)}$ Recently, minimal LAV (LAVmin) has been suggested as a better marker for LV diastolic function and filling pressure than LAVmax and has shown prognostic implications over LAVmax. ${ }^{20,21)}$ However, there are limited data on the prognostic value of LAVmin in patients with HCM. Thus, we investigated the prognostic role of LAVmin in patients with non-obstructive HCM and tested if LAVmin is better for predicting clinical outcome than LAVmax in these patients.

From the ${ }^{1}$ Division of Cardiology, Department of Internal Medicine, Inha University Hospital, Incheon, South Korea.

This work was supported by Inha University Research Grant (INHA-54769).

Address for correspondence: Sung-Hee Shin, MD, Division of Cardiology, Department of Internal Medicine, Inha University College of Medicine, 7-206, 3GA, Shinheung-Dong, Jung-Gu, Incheon, 400-711, South Korea. E-mail: sshin@inha.ac.kr

Received for publication October 23, 2017. Revised and accepted December 20, 2017.

Released in advance online on J-STAGE August 29, 2018.

doi: 10.1536/ihj.17-606

All rights reserved by the International Heart Journal Association. 


\section{Methods}

Study population: A total of 167 consecutive patients (age $=64.7 \pm 13.5$ years, male: female $=120: 47)$ who were diagnosed with non-obstructive $\mathrm{HCM}$ at our institution from 2012 to 2015 were enrolled in this study. Diagnosis of HCM was made by echocardiography based on a hypertrophied and non-dilated LV with wall thickness in the absence of other cardiac or systemic conditions that can produce a similar magnitude of hypertrophy. ${ }^{1,2)} \mathrm{Pa}$ tients with known HCM phenocopies such as Fabry disease or amyloidosis were excluded. ${ }^{22)}$ LVOT obstruction was defined as a peak instantaneous Doppler LV outflow tract pressure gradient $\geq 30 \mathrm{mmHg}$ at rest or during the Valsalva maneuver. Patients were excluded if they had significant intrinsic valvular heart disease, LV ejection fraction $\leq 45 \%$, or poor echo window. Clinical outcome included hospitalization for HF, stroke, or death. Hospitalization for $\mathrm{HF}$ was defined as an unplanned intravenous treatment of new or worsened HF with inotropic agents or diuretics requiring admission during follow-up.

Echocardiographic analysis: Echocardiographic parameters including M-mode, two-dimensional, and Doppler measurements were measured with an offline analysis workstation in accordance with the American Society of Echocardiography guidelines. ${ }^{23)}$ LAV was calculated using the disk summation algorithm and indexed to body surface area. When acquiring images to measure LAV, care was taken to avoid foreshortening of the left atrium (LA). The LA endocardial border was traced in both the apical 4- and 2-chamber views. The pulmonary veins and LA appendage were excluded from the LAV measurement. LAVmax was measured just before the mitral valve opening at the end of LV systole and LAVmin was measured at the closure of the mitral valve during end-diastole. LA volumes were normalized to body surface area to calculate the LAV index. Pulse wave Doppler was performed in the apical 4-chamber view to obtain peak early (E) and late (A) diastolic filling velocities and deceleration time of $\mathrm{E}$ wave at the tip of the mitral valve leaflets. In 25 patients with $\mathrm{AF}$ at the time of echocardiography, A velocities were not available. Tissue Doppler measurements including early mitral annular velocity (e') were derived from the septal mitral annulus.

Statistical analysis: Data are expressed as mean \pm SD for continuous variables and as count and percentage for categorical variables. Student's $t$ test or chi square test was used to compare the parameters between the groups as needed. Cox proportional hazard regression models were used to assess predictive values of clinical and echocardiographic measurements. Receiver operating characteristic (ROC) curves were constructed after logistic regression. We used the two linear predictors to get a test of the differences in area under the curves (AUC), and confidence intervals $(\mathrm{CI})$ for AUC were calculated to compare the predictive values of the models including LAVmax and LAVmin. For all tests, $P$ values were determined using 2-tailed test, and $P<0.05$ was considered to be significant. All statistical analyses were performed using STATA 14.0 (STATA Corp., College Station, TX, USA).
Table I. Baseline Characteristics

\begin{tabular}{lc}
\hline Variable & \\
\hline Age, years & $64.7 \pm 13.5$ \\
Female, $n(\%)$ & $47(28 \%)$ \\
Hypertension, $n(\%)$ & $67(40 \%)$ \\
Diabetes, $n(\%)$ & $42(25 \%)$ \\
Atrial fibrillation, $n(\%)$ & $34(20 \%)$ \\
$\quad$ Paroxysmal AF, $n(\%)$ & $9(5 \%)$ \\
Persistent/Permanent AF, $n(\%)$ & $25(15 \%)$ \\
Symptom, $n(\%)$ & \\
Asymptomatic & $83(50 \%)$ \\
Chest pain & $29(17 \%)$ \\
Palpitation & $8(5 \%)$ \\
Dizziness & $3(2 \%)$ \\
Syncope & $3(2 \%)$ \\
NYHA functional class & \\
I & $119(71 \%)$ \\
$\quad$ II & $39(23 \%)$ \\
$\quad$ III/IV & $9(5 \%)$ \\
Medication, $n(\%)$ & \\
$\beta$ blockers & $99(59 \%)$ \\
ACE inhibitor or ARB & $53(32 \%)$ \\
Calcium channel blocker & $48(29 \%)$ \\
Diuretics & $35(21 \%)$ \\
Antiarrhythmic drugs & $7(4 \%)$ \\
Antiplatelet agents & $35(21 \%)$ \\
Anticoagulant & $31(19 \%)$ \\
Statin & $56(34 \%)$ \\
\hline ACE &
\end{tabular}

ACE indicates an angiotensin converting enzyme; $\mathrm{AF}$, atrial fibrillation; $\mathrm{ARB}$, angiotension receptor blocker; and NYHA, New York Heart Association.

\section{Results}

Baseline characteristics: Table I shows the baseline characteristics of the patients. The mean age of the 167 patients was $65 \pm 14$ years $(120$ men). Among them, 67 patients had hypertension, 42 patients had diabetes and 34 patients had AF. Half of the patients in our study population were asymptomatic while the most common symptom was dyspnea. The mean LV ejection fraction was $64.6 \pm$ $5.0 \%$ and maximal and minimal LAV indexes were $43.6 \pm$ $18.1 \mathrm{~mL} / \mathrm{m}^{2}$ and $25.2 \pm 17.3 \mathrm{~mL} / \mathrm{m}^{2}$, respectively.

Predictors for clinical events and mortality: During a median follow-up of $25.0 \pm 17.8$ months, 35 patients $(21 \%)$ experienced clinical events, of which 8 patients (5\%) died, 9 patients $(5 \%)$ were hospitalized for $\mathrm{HF}$, and 18 patients $(11 \%)$ had a stroke. As shown in Table II, patients with clinical events were older and had a higher prevalence of AF than patients without events (all $P$ values $<0.001)$. Gender and other comorbidities such as hypertension and diabetes were comparable between the groups. In addition, the maximal and minimal LA volumes were larger and E/e'was higher in patients with clinical events than in those without events (all $P$ values < 0.001) whereas LV dimension, LV systolic function, maximal wall thickness, and other Doppler parameters were not significantly different (Table II).

Cox regression analysis also identified age, AF, E/e', and both maximal and minimal LA volumes as univariate associates of clinical events. In multivariate analysis, 
Table II. Univariate Relations of Clinical and Echocardiographic Parameters with Clinical Outcomes

\begin{tabular}{lccc}
\hline & $\begin{array}{c}\text { Patients without events } \\
(n=132)\end{array}$ & $\begin{array}{c}\text { Patients with events } \\
(n=35)\end{array}$ & $P$ value \\
\hline Clinical factors & & & \\
Age, years & $62.8 \pm 13.4$ & $71.8 \pm 11.9$ & $<0.001^{*}$ \\
Female, $n(\%)$ & $33(25 \%)$ & $14(40 \%)$ & 0.08 \\
Hypertension & $50(38 \%)$ & $17(49 \%)$ & 0.25 \\
Diabetes & $33(25 \%)$ & $9(26 \%)$ & 0.93 \\
AF & $18(14 \%)$ & $16(46 \%)$ & $<0.001^{*}$ \\
Echocardiographic parameters & & & \\
LVEDD index, mm/m ${ }^{2}$ & $26.6 \pm 3.1$ & $27.0 \pm 3.5$ & 0.58 \\
LVESD index, mm/m & $16.5 \pm 2.5$ & $16.8 \pm 2.6$ & 0.56 \\
LVEF, \% & $64.7 \pm 4.3$ & $64.3 \pm 5.2$ & 0.61 \\
LAVmax index, $\mathrm{mL} / \mathrm{m}^{2}$ & $39.2 \pm 14.1$ & $59.8 \pm 21.9$ & $<0.001^{*}$ \\
LAVmin index, mL/m ${ }^{2}$ & $20.6 \pm 12.6$ & $42.5 \pm 21.3$ & $<0.001^{*}$ \\
Maximal wall thickness, mm & $20.0 \pm 4.1$ & $20.0 \pm 3.3$ & 0.98 \\
E, cm/second & $65.5 \pm 20.4$ & $72.4 \pm 20.9$ & 0.08 \\
A, cm/second & $130.5 \pm 55.7$ & $74.9 \pm 21.0$ & 0.65 \\
E/A & $0.95 \pm 0.43$ & $0.95 \pm 0.52$ & 0.99 \\
DT, mseconds & $255.8 \pm 69.9$ & $246.5 \pm 76.3$ & 0.50 \\
E/e' & $13.5 \pm 5.2$ & $17.6 \pm 7.3$ & $<0.001^{*}$ \\
\hline
\end{tabular}

A indicates peak late mitral inflow velocity; DT, deceleration time; E, peak late mitral inflow velocity; e', mitral annular velocity; LAVmax, maximal left atrial volume; LAVmin, minimal left atrial volume; LVEDD, left ventricular end-diastolic dimension; LVEF, left ventricular ejection fraction; and LVESD, left ventricular end-systolic dimension. ${ }^{*} P<0.05$

Table III. Hazard Rratios (95\% CIs) for Composite Outcome in Univariate and Multivariate Cox Models

\begin{tabular}{lccccccccc}
\hline & \multicolumn{3}{c}{ Univariate } & \multicolumn{3}{c}{ Multivariate (1) } & \multicolumn{3}{c}{ Multivariate (2) } \\
& HR & $95 \%$ CI & $P$ value & HR & $95 \%$ CI & $P$ value & HR & $95 \%$ CI & $P$ value \\
\hline Age & 1.06 & $1.01-1.13$ & $0.02^{*}$ & 1.04 & $0.99-1.11$ & 0.14 & 1.03 & $0.98-1.10$ & 0.24 \\
AF & 3.06 & $1.13-8.30$ & $0.03 *$ & 1.21 & $0.39-3.75$ & 0.74 & 0.86 & $0.24-3.04$ & 0.81 \\
E/e' & 1.09 & $1.02-1.15$ & $0.01 *$ & 1.03 & $0.97-1.10$ & 0.36 & 1.03 & $0.98-1.11$ & 0.22 \\
LAVmax index & 1.06 & $1.03-1.08$ & $<0.001 *$ & 1.05 & $1.02-1.08$ & $0.001 *$ & & & \\
LAVmin index & 1.06 & $1.04-1.09$ & $<0.001^{*}$ & & & & 1.06 & $1.03-1.09$ & $0.001^{*}$ \\
\hline
\end{tabular}

AF indicates atrial fibrillation; $\mathrm{E}$, peak late mitral inflow velocity; e', mitral annular velocity; LAVmax, maximal left atrial volume; and LAVmin, minimal left atrial volume. $* P<0.05$

maximal and minimal LA volumes were independently associated with a worse outcome (Table III). To avoid collinearity, we constructed 2 different multivariate models using LAVmax and LAVmin separately and compared them. The AUC calculated by ROC analysis for predicting clinical outcome was significantly larger in the model including LAVmin than the model including LAVmax (AUC for model 1 including age, AF, LV ejection fraction, E/e', and indexed maximal LA volume $=0.82[95 \% \mathrm{CI}=0.73$ 0.90], AUC for model 2 including age, AF, LV ejection fraction, E/e', and indexed minimal LA volume $=0.85$ [95\% CI $=0.77-0.93], P=0.02$, Figure).

\section{Discussion}

HCM is characterized by a diverse phenotypic expression and clinical course. LVOT obstruction is an independent predictor of morbidity and mortality in patients with HCM. ${ }^{6,7)}$ However, nonobstructive HCM has more heterogeneous disease spectrum, and its clinical course is not as well understood. While non-obstructive HCM is reported to have a relatively benign clinical course, several studies have shown that significant proportion of these patients experience HCM related morbidity and mortality. $^{8-10,24)}$ In our study, $21 \%$ of the patients with nonobstructive HCM experienced hospitalization for HF, stroke, or death during monitoring, suggesting that these patients should be followed up with caution. In predicting clinical outcome, both maximal and minimal LA volumes were independent factors even after adjusting for clinical and echocardiographic parameters.

The LA can act as a reservoir during LV systole, a passive conduit during early diastole, and an active contractile chamber during late diastole. ${ }^{25)} \mathrm{LA}$ enlargement is common in patients with HCM and LA remodeling is associated with multifactorial conditions such as diastolic function, intrinsic myocardial stiffness, and rhythm disturbance. Severity of LV hypertrophy, LVOT obstruction, and mitral regurgitation can also be attributed to LA dilatation. Several studies have shown that LA volume can be related to disease severity and functional capacity as well as clinical outcome in HCM. ${ }^{16,17,26)}$ As a continuum of the LV, LA size and function can be influenced by LV function. Numerous studies have shown the value of LA size in vari- 


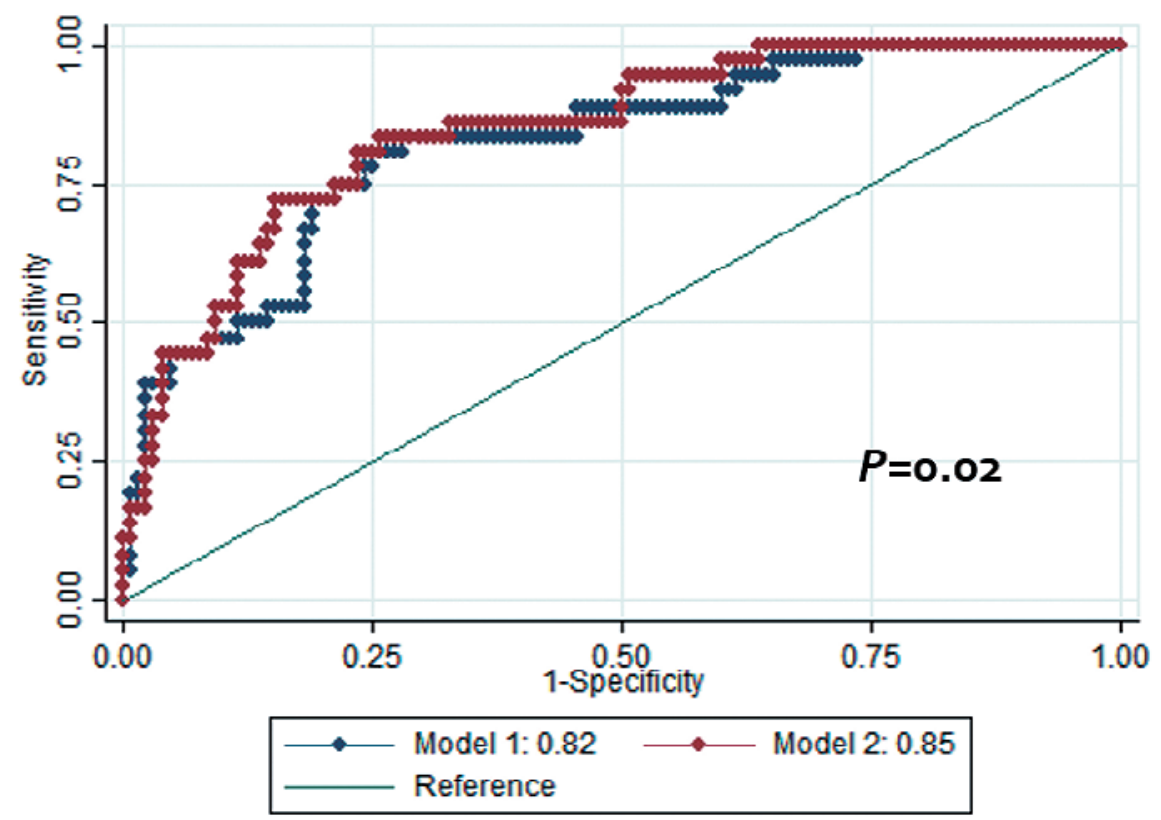

Figure. Receiver operating characteristic curves of the models including maximal LAV or minimal LAV in predicting clinical outcome. Model 1 included age, atrial fibrillation, left ventricular ejection fraction, E/e', and indexed maximal left atrial volume. Model 2 included age, atrial fibrillation, left ventricular ejection fraction, E/e', and indexed minimal left atrial volume.

ous clinical settings and volumetric assessment is known to be more accurate than linear assessment for LA size. In general, LA volume is measured at the LV end-systolic phase, when it is the greatest. Maximal LA volume can reflect the severity and chronicity of elevated LV filling pressure and has been suggested as a potential biomarker and prognosticator for cardiac diseases. However, maximal LAV can be influenced by LV systolic function through longitudinal systolic motion of the mitral annulus. ${ }^{19,27)}$ Systolic descent of the mitral annular plane toward the LV apex can stretch the LA, which leads to active suction of blood into the LA from the pulmonary veins. Thus, LV longitudinal systolic function can affect maximal LAV. On the other hand, minimal LAV, which is measured at the LV end-diastolic phase, is directly exposed to LV enddiastolic pressure and theoretically can be a better marker than maximal LAV. Prior studies have shown that minimal LAV was related to elevated pulmonary wedge pressure in patients undergoing cardiac catheterization, suggesting that it is a better correlate of LV diastolic function than maximal LAV. ${ }^{20,28,29)}$ While both maximal and minimal LA volumes increased with LV diastolic dysfunction, the change in minimal LAV was more pronounced. ${ }^{20)}$ Additionally, several studies have demonstrated that LAVmin was associated with clinical events. LAVmin was more predictive of the first AF or flutter in an elderly cohort and can provide more relevant information on prognosis than LAVmax. ${ }^{21,30,31)}$ In our study, minimal LAV was a better predictor of worse outcome than maximal LAV in patients with non-obstructive HCM. This measure of LA remodeling may provide chronic diastolic burden more reliably in this population. Further studies with a larger general population are necessary to generalize our results.
Some limitations of our study should be noted. The presence of dynamic LVOT obstruction itself is known to be related to disease morbidity and mortality. While we enrolled patients with non-obstructive HCM in our study, we defined LVOT obstruction using the Doppler method at rest and during the Valsalva maneuver. We did not perform exercise stress echocardiography in symptomatic patients with an LVOT gradient $<50 \mathrm{mmHg}$, so some patients with provoked LVOT obstruction might be included in our data.

Second, we assessed LAV using two-dimensional echocardiography. The accuracy of this method can be intrinsically limited because of its dependency on the angle and geometric assumption of the LA shape. Prior studies have shown that three-dimensional (3D) echocardiography is more accurate for LAV compared with the cardiac magnetic resonance imaging reference and has better prognostic utility than 2D echocardiography. However, we did not use $3 \mathrm{D}$ echocardiography for assessing LAV in our population since many patients had AF, which limited the use of $3 \mathrm{D}$ echocardiography. Instead, we attempted to avoid foreshortening of the LA images from both apical views to minimize errors that can occur during 2D assessment. Finally, study subjects were recruited from a single referral tertiary center. Thus, our study population might not be representative of the general population with nonobstructive HCM. Rather, our population might be a skewed selected population with regard to disease severity and comorbidities.

\section{Conclusion}

In patients with non-obstructive HCM, both maximal 
and minimal LA volumes were independent predictors of $\mathrm{HF}$, stroke, or death even after adjusting for clinical and echocardiographic factors. Minimal LAV was a better predictor of worse outcome than was maximal LAV in our study. These results suggest that minimal LAV can be a more reliable marker for prognosis in this population.

\section{Disclosures}

Conflicts of interest: None.

\section{References}

1. Gersh BJ, Maron BJ, Bonow RO, et al. American Association for Thoracic Surgery; American Society of Echocardiography; American Society of Nuclear Cardiology; Heart Failure Society of America; Heart Rhythm Society; Society for Cardiovascular Angiography and Interventions. Circulation ACCF/AHA: Society of Thoracic Surgeons 2011; 124: e783-831.

2. Authors/Task Force members, Elliott PM, Anastasakis A, et al. 2014 ESC Guidelines on diagnosis and management of hypertrophic cardiomyopathy: the Task Force for the Diagnosis and Management of Hypertrophic Cardiomyopathy of the European Society of Cardiology (ESC). Eur Heart J 2014; 35: 2733 79.

3. Kubo T, Baba Y, Hirota T, et al. Prognostic significance of nondilated left ventricular size and mitral regurgitation in patients with dilated phase of hypertrophic cardiomyopathy. Int Heart J 2017; 58: 63-8.

4. Maron BJ, Casey SA, Poliac LC, Gohman TE, Almquist AK, Aeppli DM. Clinical course of hypertrophic cardiomyopathy in a regional United States cohort. JAMA 1999; 281: 650-5.

5. Elliott PM, Gimeno JR, Thaman R, et al. Historical trends in reported survival rates in patients with hypertrophic cardiomyopathy. Heart 2006; 92: 785-91.

6. Maron MS, Olivotto I, Betocchi S, et al. Effect of left ventricular outflow tract obstruction on clinical outcome in hypertrophic cardiomyopathy. N Engl J Med 2003; 348: 295-303.

7. Autore C, Bernabò $\mathrm{P}$, Barillà CS, Bruzzi $\mathrm{P}$, Spirito $\mathrm{P}$. The prognostic importance of left ventricular outflow obstruction in hypertrophic cardiomyopathy varies in relation to the severity of symptoms. J Am Coll Cardiol 2005; 45: 1076-80.

8. Maron MS, Rowin EJ, Olivotto I, et al. Contemporary natural history and management of nonobstructive hypertrophic cardiomyopathy. J Am Coll Cardiol 2016; 67: 1399-409.

9. Maron BJ, Rowin EJ, Maron MS, Braunwald E. Nonobstructive hypertrophic cardiomyopathy out of the shadows: known from the beginning but largely ignored ... until now. Am J Med 2017; 130: 119-23.

10. Moon J, Shim CY, Ha JW, et al. Clinical and echocardiographic predictors of outcomes in patients with apical hypertrophic cardiomyopathy. Am J Cardiol 2011; 108: 1614-9.

11. Tsang TS, Barnes ME, Gersh BJ, et al. Prediction of risk for first age-related cardiovascular events in an elderly population: the incremental value of echocardiography. J Am Coll Cardiol 2003; 42: 1199-205.

12. Moller JE, Hillis GS, Oh JK, et al. Left atrial volume: a powerful predictor of survival after acute myocardial infarction. Circulation 2003; 107: 2207-12.

13. Rossi A, Cicoira M, Zanolla L, et al. Determinants and prognostic value of left atrial volume in patients with dilated cardiomyopathy. J Am Coll Cardiol 2002; 40: 1425.

14. Losi MA, Betocchi S, Aversa M, et al. Determinants of atrial fibrillation development in patients with hypertrophic cardiomyopathy. Am J Cardiol 2004; 94: 895-900.

15. Tani T, Tanabe K, Ono M, et al. Left atrial volume and the risk of paroxysmal atrial fibrillation in patients with hypertrophic cardiomyopathy. J Am Soc Echocardiogr 2004; 17: 644-8

16. Kjaergaard J, Johnson BD, Pellikka PA, Cha SS, Oh JK, Ommen SR. Left atrial index is a predictor of exercise capacity in patients with hypertrophic cardiomyopathy. J Am Soc Echocardiogr 2005; 18: 1373-80.

17. Sachdev V, Shizukuda Y, Brenneman CL, et al. Left atrial volumetric remodeling is predictive of functional capacity in nonobstructive hypertrophic cardiomyopathy. Am Heart J 2005; 149: 730-6.

18. Losi MA, Betocchi S, Barbati G, et al. Prognostic significance of left atrial volume dilatation in patients with hypertrophic cardiomyopathy. J Am Soc Echocardiogr 2009; 22: 76-81.

19. Kwan J, Qin JX, Popović ZB, Agler DA, Thomas JD, Shiota T. Geometric changes of mitral annulus assessed by real-time 3dimensional echocardiography: becoming enlarged and less nonplanar in the anteroposterior direction during systole in proportion to global left ventricular systolic function. J Am Soc Echocardiogr 2004; 17: 1179-84.

20. Russo C, Jin Z, Homma S, et al. Left atrial minimum volume and reservoir function as correlates of left ventricular diastolic function: impact of left ventricular systolic function. Heart 2012; 98: 813-20.

21. Wu VC, Takeuchi M, Kuwaki H, et al. Prognostic value of la volumes assessed by transthoracic 3D echocardiography: comparison with 2D echocardiography. JACC Cardiovasc Imaging 2013; 6: 1025-35.

22. Anan R. Fabry disease and/or hypertrophic cardiomyopathy. Int Heart J 2017; 58: 305-6.

23. Lang RM, Badano LP, Mor-Avi V, et al. Recommendations for cardiac chamber quantification by echocardiography in adults: an update from the American Society of Echocardiography and the European Association of Cardiovascular Imaging. Eur Heart J Cardiovasc Imaging 2015; 16: 233-70.

24. Siontis KC, Geske JB, Ong K, Nishimura RA, Ommen SR, Gersh BJ. Atrial fibrillation in hypertrophic cardiomyopathy: prevalence, clinical correlations, and mortality in a large highrisk population. J Am Heart Assoc 2014; 3: e001002.

25. Blume GG, Mcleod CJ, Barnes ME, et al. Left atrial function: physiology, assessment, and clinical implications. Eur J Echocardiogr 2011; 12: 421-30.

26. Yang H, Woo A, Monakier D, et al. Enlarged left atrial volume in hypertrophic cardiomyopathy: a marker for disease severity. J Am Soc Echocardiogr 2005; 18: 1074-82.

27. Barbier P, Solomon SB, Schiller NB, Glantz SA. Left atrial relaxation and left ventricular systolic function determine left atrial reservoir function. Circulation 1999; 100: 427-36.

28. Hedberg P, Selmeryd J, Leppert J, Henriksen E. Left atrial minimum volume is more strongly associated with N-terminal proB-type natriuretic peptide than the left atrial maximum volume in a community-based sample. Int J Cardiovasc Imaging 2016; 32: 417-25.

29. Appleton CP, Galloway JM, Gonzalez MS, Gaballa M, Basnight MA. Estimation of left ventricular filling pressures using twodimensional and Doppler echocardiography in adult patients with cardiac disease. Additional value of analyzing left atrial size, left atrial ejection fraction and the difference in duration of pulmonary venous and mitral flow velocity at atrial contraction. J Am Coll Cardiol 1993; 22: 1972-82.

30. Fatema K, Barnes ME, Bailey KR, et al. Minimum vs. maximum left atrial volume for prediction of first atrial fibrillation or flutter in an elderly cohort: a prospective study. Eur J Echocardiogr 2009; 10: 282-6.

31. Caselli S, Canali E, Foschi ML, et al. Long-term prognostic significance of three-dimensional echocardiographic parameters of the left ventricle and left atrium. Eur J Echocardiogr 2010; 11: 250-6. 